\title{
APORTES A LA CONSERVACIÓN DE Sternula lorata, "GAVIOTÍN PERUANO”, EN LA RESERVA NACIONAL DE PARACAS (RNP), ICA - PERÚ
}

\section{CONTRIBUTIONS TO THE CONSERVATION OF Sternula lorata, PERUVIAN TERN, AT PARACAS NATIONAL RESERVE (PNR), ICA - PERU}

\author{
Samuel Amorós ${ }^{1}$ y Patricia Saravia ${ }^{2}$
}

\begin{abstract}
Resumen
Sternula lorata está gravemente amenazada y su población mundial se estima de 1000 a 2499 individuos. La investigación se realizó en la península de Paracas donde se registró un máximo de 605 individuos además de 37 nidos durante la temporada reproductiva 2006 - 2007. Ambos valores superan los reportados para otras localidades y resaltan la importancia de esta península para la conservación de esta ave.

Las amenazas naturales de esta población estuvieron representadas por depredadores potenciales como: Pseudalopex sp., “Zorro”; Falco peregrinus, "Halcón peregrino”; Buteo polyosoma, “Aguilucho común”; y Cathartes aura, "Gallinazo cabeza roja”. Por otro lado, las amenazas antrópicas incluyeron la propuesta de desarrollo de actividades no compatibles con la conservación del lugar y el tránsito vehicular. En base a estos hallazgos se recomienda el desarrollo de estrategias participativas para lograr la conservación de esta especie amenazada al interior de la península de Paracas, así como también sumar esfuerzos para su conservación a lo largo de todo el ámbito de su distribución.
\end{abstract}

Palabras clave: Conservación y amenazas, Gaviotín Peruano, Paracas, Sternula lorata.

\begin{abstract}
Sternula lorata is an endangered species. Worldwide, it is estimated that only from 1000 to 2499 individuals are left. This research was carried out in the Paracas Peninsula where a maximum of 605 individuals and 37 active nests were found during the reproductive season of 2006-2007. Both values surpass the reports for other localities and highlight the importance of this peninsula for the conservation of this species.

Natural threats were represented by potential predators such as: Pseudalopex sp., "Fox"; Falco peregrinus, "Peregrine falcon"; Buteo polyosoma, "Variable hawk" and Cathartes aura, "Turkey vulture". On the other hand, the development of activities that are not compatible with the conservation of the area and vehicular traffic, were identified as threats of great negative impact. These findings lead us to recommend that participatory strategies should be developed to attain the conservation of this threatened species within the Paracas Peninsula and throughout the entire area of this species distribution.
\end{abstract}

Key words: conservation and threats, Paracas, Peruvian Tern, Sternula lorata.

\section{Introducción.}

Sternula lorata "gaviotín peruano" es una especie con graves amenazas para su conservación. Entre estas destacan la pérdida del hábitat y las perturbaciones en los lugares de reproducción (BirdLife International, 2011). Su población está disminuyendo, habiendo sido estimada en 10000 individuos (Duffy et al., 1984). Actualmente su población mundial se calcula entre 1000 y 2499 individuos (BirdLife International, 2011) por lo que se encuentra categorizada como especie "En Peligro" (Endangered, en; BirdLife International, 2011) y en el Perú como especie vulnerable (Decreto Supremo No 034-2004-AG).

$S$. lorata es una especie endémica de la corriente de Humboldt (Vilina, 1998), se distribuye desde La
Isla El Muerto en el Golfo de Guayaquil en Ecuador hasta Antofagasta en Chile (Murphy, 1936; Harrison 1985; Vilina 1998) y su área de reproducción se encuentra a lo largo del litoral peruano (Murphy, 1936; Harrison, 1985) y el litoral chileno hasta Mejillones - Región de Antofagasta (Vilina, 1998). En el Perú se conocen cuatro lugares de reproducción: Pacasmayo, Paraíso, península de Paracas y Tres Hermanas-Yanyarina; mientras que en Chile se conocen ocho lugares, todos ubicados en la península de Mejillones (Guerra-Correa, 2003; Zavalaga et al., 2009 y Amorós et al., 2010).

El presente trabajo aporta al conocimiento del estado de conservación de $S$. lorata al interior de la península de Paracas mediante la evaluación de la 
abundancia de esta especie y las amenazas que presenta al interior de la colonia reproductiva. Asimismo, compara la abundancia de individuos y número de nidos hallados en dicha península con los reportados en otros lugares donde la especie se distribuye y/o reproduce. Esta información permitirá elaborar estrategias que garanticen la conservación de esta ave amenazada al interior de la península de Paracas. Adicionalmente, se presenta el registro de otras especies de aves que comparten el hábitat reproductivo con $S$. lorata.

Materiales y métodos. Área de estudio. junio 2007 y julio 2008 se realizaron salidas ocasionales.

Determinación del número de individuos.

Se registró la abundancia del $S$. lorata basados en el método del conteo directo (Rodríguez, 1987; Velarde, 1998). Cuando fue posible, se diferenció el grupo en dos clases de edades: juveniles y adultos. El número total de individuos fue registrado cada mes desde un punto fijo de observación y con el uso de binoculares. Las observaciones fueron realizadas por uno o más observadores y se tomó el valor promedio. Durante las primeras salidas se identificó el lugar donde se realizaría la observación, la extensión del área observada así como los periodos en los cuales

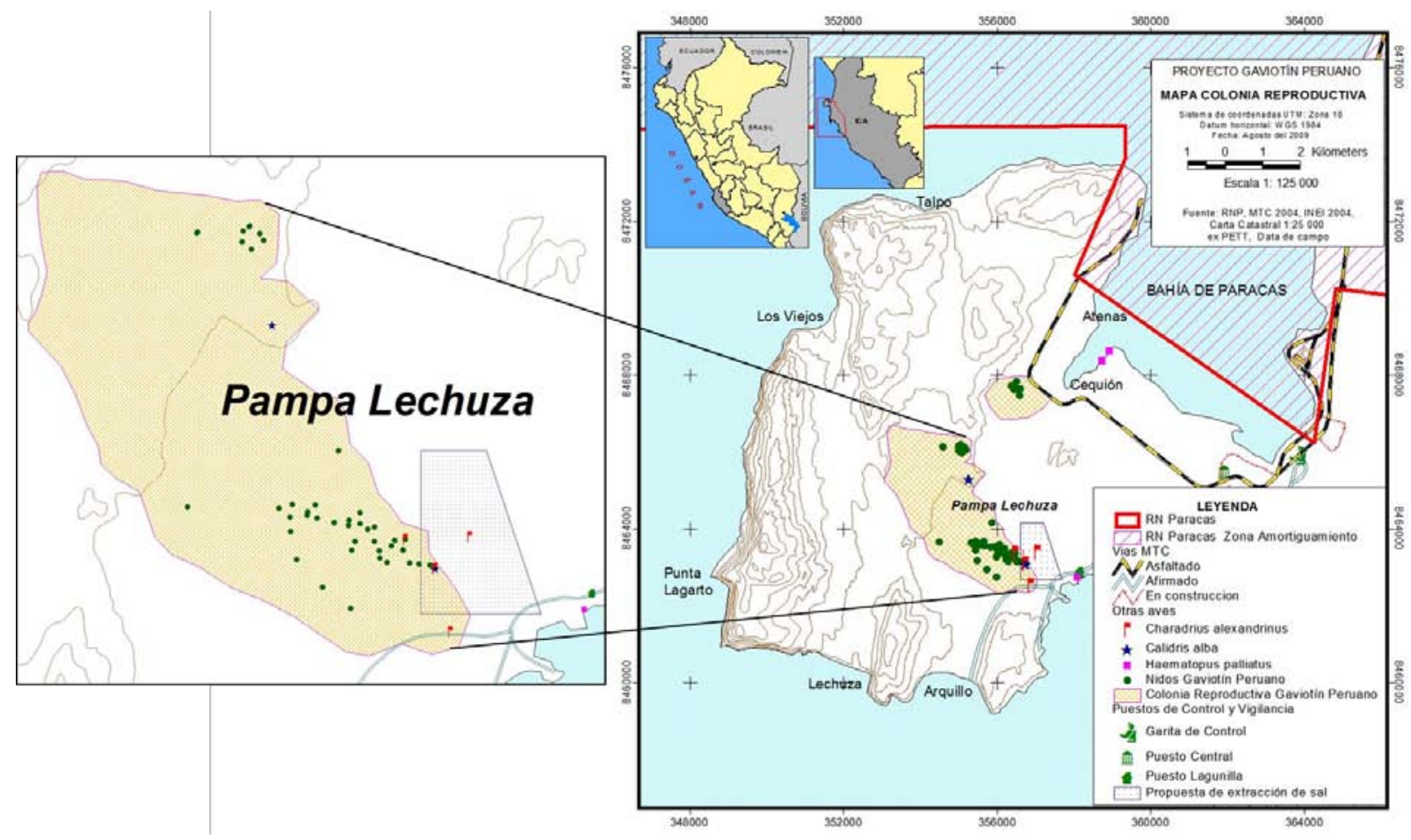

Figura 1. Colonia reproductiva de Sternula lorata, "Gaviotín peruano”.

El estudio fue desarrollado en la colonia reproductiva de $S$. lorata encontrada en Pampa Lechuza y en Pampa Atenas - Cequión (Amorós et al., 2010), al interior de la península de Paracas, ubicada en la zona norte de la Reserva Nacional de Paracas - RNP (Figura 1). La RNP se encuentra entre los paralelos $13^{\circ} 47^{\prime} \mathrm{S}$ y $14^{\circ} 26^{\prime} \mathrm{S}$ y los $76^{\circ} 30^{\prime} \mathrm{O}$ y los $76^{\circ} 00^{\prime} \mathrm{O}$ y políticamente se ubica en el departamento de Ica, provincia de Pisco e Ica, distritos de Paracas y Salas, respectivamente. Esta área natural protegida presenta 216 especies de aves y $S$. lorata constituye un objeto de conservación de la misma (INRENA, 2002). El trabajo de campo se realizó en forma sistemática desde noviembre 2006 hasta mayo 2007 mediante salidas de campo de 2 a 3 días de duración y en grupos de 2 a 4 personas. Las salidas de campo se realizaron cada 15 días aproximadamente. Adicionalmente, entre realizar los conteos a lo largo del estudio.

Estas observaciones se realizaron desde un lugar en el cual se podía observar la mayor parte de la zona de reproducción de la especie en estudio. Asimismo se encontró que $S$. lorata se concentraba en dicho espacio utilizándolo como dormidero o lugar de descanso. El área aproximada de observación fue de unas 2 ha y fuera de esta zona no se han observado otros lugares en el desierto con altas concentraciones de $S$. lorata. Inicialmente se realizaron conteos directos durante todo el día, mientras se realizó el estudio de biología reproductiva (Amorós et al., 2010).

También se registró el encuentro ocasional de otras especies de aves que comparten el hábitat con $S$. lorata y que aparentemente no representan amenazas para su conservación. En el caso de encontrar nidos de estas especies, se midió el diámetro mayor, diámetro 
menor y profundidad con una regla y/o cinta métrica (wincha) ( $\pm 1 \mathrm{~mm}$ ); se registraron sus coordenadas con un navegador GPS y se describieron sus principales características.

Identificación de las amenazas.

Se registraron de forma cualitativa las amenazas observadas en el campo que afectan o podrían afectar a $S$. lorata en su colonia reproductiva. Las amenazas fueron agrupadas en naturales y antrópicas según las características que las causaron. En el primer grupo se registraron los depredadores observados directa o indirectamente (huellas) en la colonia reproductiva; mientras que en el segundo grupo se identificaron aquellas actividades humanas que tienen un alto potencial de impactar negativamente la colonia reproductiva de $S$. lorata. encuentran: 1) Calidris alba, "playerito blanco", el cual utiliza el desierto como lugar de descanso; 2) Charadrius alexandrinus, "chorlo nevado", que se reproduce en la periferia de la colonia de S. lorata; y también usa el desierto como lugar de descanso 3) Haematopus palliatus, "ostero común”, se reproduce cerca de las orillas de playa; y 4) Oceanodroma markhami, "golondrina negra de la tempestad", que presenta colonias reproductivas en determinadas zonas de pendiente de la península de Paracas.

Se encontraron tres nidos activos de Charadrius alexandrinus. Uno de ellos con 3 huevos se encontró a $1.5 \mathrm{~m}$ aproximadamente de un nido activo de $S$. lorata en la periferia de la colonia reproductiva de Pampa Lechuza. El otro, también con 3 huevos, ubicado en la zona sur de la misma colonia y a aproximadamente

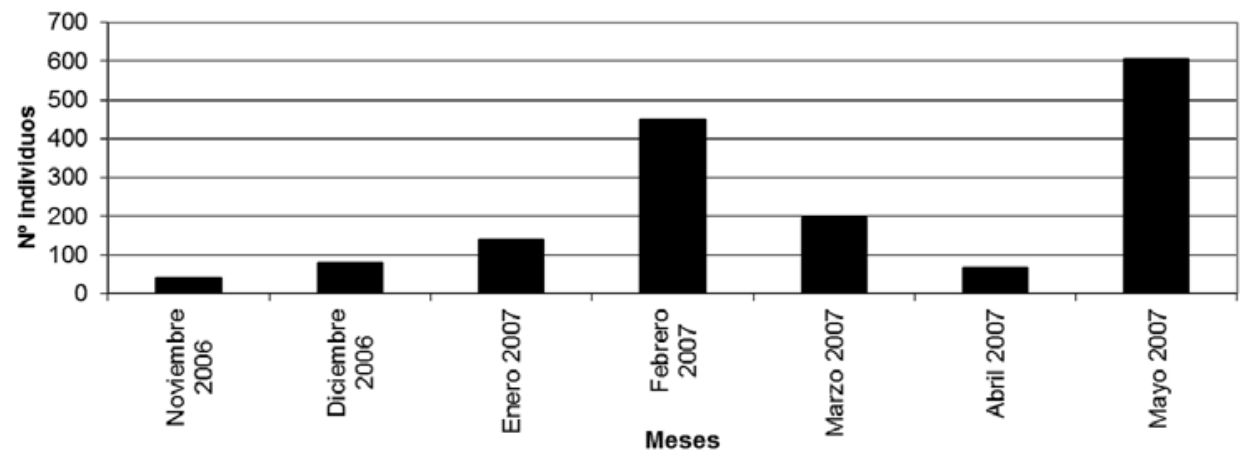

Figura 2. Conteo de Sternula lorata, "Gaviotín peruano" (adultos y juveniles), en la península de Paracas (RNP, Ica - Perú) entre la temporada reproductiva 2006-2007.

\section{Resultados.}

Determinación del número de individuos y periodos de actividad.

Se registró un total de 605 individuos que representa el $24.2 \%$ a $60.5 \%$ de la población mundial de esta especie. El mayor número de individuos se encontró en el mes de mayo del 2007, el cual correspondió a 550 individuos adultos y 55 individuos juveniles. Generalmente los individuos fueron observados reunidos en una 0 dos bandadas provenientes de direcciones distintas y las cuales se unían en los alrededores del punto de observación. Los valores máximos mensuales pueden observarse en la Figura 2.

S. lorata presenta mayor actividad entre las 6:00 a.m. y las 9:00 a.m., periodo del día donde podía observarse una mayor abundancia de individuos. Luego de este periodo prácticamente desaparece del desierto y/o tan sólo se pueden ver unos 4 a 6 individuos volando por los alrededores o algunos otros posados sobre sus nidos.

Encuentro ocasional con otras especies de aves.

Se observó que $S$. lorata comparte el hábitat con otras aves cuya presencia aparentemente no constituye una amenaza para su conservación. Entre estas se
940 m de distancia más cercana a un nido activo de la especie en estudio. Finalmente el tercer nido, con un huevo, se ubicó al interior del lugar donde se establecería el lote para la extracción de sal. Los nidos de Charadrius alexandrinus se caracterizaron por presentar decoración de pequeñas piedras en el interior y rodeando a este. Las dimensiones de uno de estos nidos fue de $16 \mathrm{~cm}$ de diámetro mayor, $10.5 \mathrm{~cm}$ de diámetro menor y $2.5 \mathrm{~cm}$ de profundidad (Figura 3a).

Se encontraron cuatro nidos de Haematopus palliatus de los cuales tres eran nidos activos. Dos presentaron tres huevos cada uno y el otro presentó dos pichones, mientras que el cuarto fue un nido abandonado. Los nidos de Haematopus palliatus también se caracterizaron por presentar decoración de pequeñas piedras en el interior y rodeando a este. En un caso se observó que presentaron conchuelas y patas de cangrejo. La medida de uno de ellos fue: $29.0 \mathrm{~cm}$ de diámetro mayor, $26.0 \mathrm{~cm}$ de diámetro menor y 2.5 cm de profundidad (Figura 3b).

Identificación de amenazas

Se identificaron las siguientes amenazas:

\section{Amenazas naturales:}

Pseudalopex sp. "zorro": En los alrededores de la colonia reproductiva y en el desierto de la península 


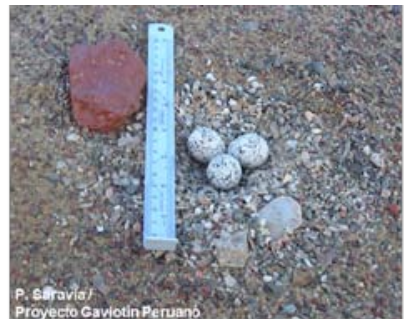

a.

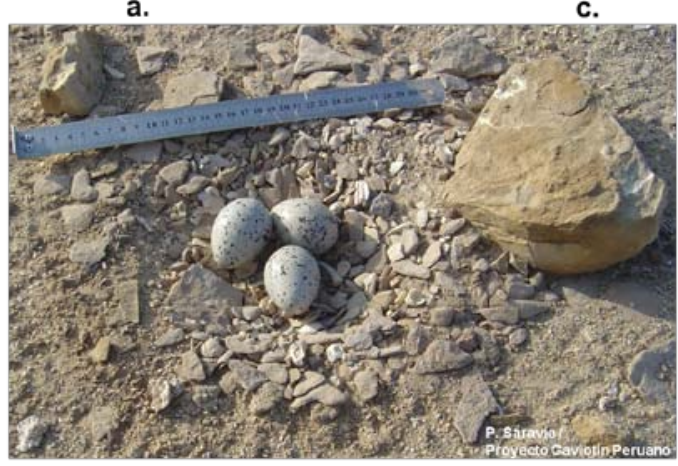

b.

Figura 3. Nido de Charadrius alexandrinus, "Chorlo nevado" (a), Haematopus palliatus, “Ostrero común” (b) y Sternula lorata, "Gaviotín peruano” (c).

de Paracas es común observar huellas de “zorro”. Sin embargo, sólo en una oportunidad se encontró un nido cuya pérdida de huevos podría haber sido causada por esta especie. En la periferia de este nido y sobre el mismo se encontraron huellas que aparentemente indicaban que el "zorro" había atacado el nido.

Falco peregrinus "halcón peregrino”: Se observó un "halcón peregrino" en dos diferentes fechas; sin embargo, en ambas oportunidades no se registró ningún intento de ataque a $S$. lorata.

Buteo polyosoma "aguilucho común”: Se observó dos "aguiluchos comunes" en tres diferentes fechas y en una de ellas el "aguilucho común” intentó atacar, sin éxito, a dos individuos adultos de $S$. lorata que se encontraban en sus respectivos nidos.

Cathartes aura "gallinazo cabeza roja": Se observó, en una oportunidad, 12 "gallinazos cabeza roja” volando por los alrededores de la colonia reproductiva. También se encontró huellas de estos en la colonia reproductiva. Sin embargo, no se ha observado consumo de huevos o pichones por parte esta especie.

\section{Amenazas antrópicas:}

Propuesta de extracción de sal: Durante el desarrollo de la investigación existió una propuesta para extraer sal de la zona de Lagunillas (Vargas y Obando, 2008) y cuyos límites se sobreponen con parte de la colonia reproductiva de $S$. lorata (Figura 1)

Tránsito vehicular: El tránsito de vehículos constituye la mayor amenaza para $S$. lorata dado el inminente riesgo de atropello de nidos, huevos y/o pichones. Se observó que al interior de la zona reproductiva y por sectores cercanos a esta existe un conjunto de vías (trochas no establecidas por el área natural protegida) cuya frecuencia de uso es variable y permiten que los diferentes actores sociales (pescadores, personal de la Reserva, turistas, investigadores, entre otros) se desplacen a distintos puntos de la península sin conocer que dicho tránsito podría poner en riesgo a $S$. lorata. Entre los vehículos que transitan por el interior de la colonia reproductiva se cuenta con: carros, motos, camionetas de turistas y ocasionalmente motociclistas de motocross (actividad realizada en forma ilegal ya que las autoridades de la RNP prohíben su uso al interior del área natural protegida). El riesgo de impacto del tránsito vehicular sobre los nidos de $S$. lorata puede observarse en la Tabla 1 donde se tiene aquellos nidos cuya distancia a las trochas es menor o igual a los $30 \mathrm{~m}$.

Tabla 1. Distancia entre nidos de Sternula lorata “Gaviotín peruano” y trochas.

\begin{tabular}{lc}
\hline Nido & Distancia de trocha al Nido $(\mathrm{m})$ \\
\hline Nido 1 & 15.0 \\
Nido 3 & 30.0 \\
Nido 5 & 1.5 \\
Nido 15 & 12.0 \\
Nido 16 & 30.0 \\
\hline
\end{tabular}

\section{Discusión.}

En el Perú, desde 1920 hasta finales de la década de los 60, el único lugar confirmado donde $S$. lorata se reproducía era Pacasmayo (Murphy, 1936). Luego, desde 1969 hasta finales de la década de los 70, se descubrieron 4 lugares más: 1) Puerto Viejo, 2) Mollendo, ambos perdidos por las perturbaciones antrópicas, 3) Pativilca; estos 3 reportados por Zavalaga et al. (2008), y 4) Tres Hermanas Yanyarina por Galarza (Galarza, 1968). Entre los años 90 y 2005 se han reportado: 1) Pampa Lechuza, 2) Yanyarina, 3) Pacasmayo; estos 3 reportados por Zavalaga et al. (2008) y 4) Paraíso por Tello et al. (2005). Actualmente en Perú se conoce sólo 4 lugares de reproducción de esta especie: Pacasmayo, Paraíso, península de Paracas, y Tres Hermanas - Yanyarina (Zavalaga et al., 2009 y Amorós et al., 2010); mientras que en Chile, se conocen 8 sitios de reproducción al interior de la península de Mejillones (Guerra-Correa, 2003). De todos ellos (Tabla 2) la península de Paracas constituye uno de los lugares con mayor número de nidos, 37 nidos en la temporada reproductiva 2006-2007 (Amorós et al., 2010); asimismo, el valor de 605 individuos observados en la península de Paracas supera al reportado para otras localidades donde la especie se distribuye (Tabla 3). Si bien los reportes históricos mencionan decenas de miles de individuos observados en el puerto de Paita 
en 1925 (Murphy, 1936), así como los 10000 individuos reportados para Perú y Chile por Duffy et al. (1984); actualmente, las estimaciones globales señalan que la población de $S$. lorata varía entre los 1000 y 2499 individuos (BirdLife International, 2011). Los estudios realizados por Zavalaga et al. (2009) reportan 283 individuos contados entre el 2003 y el 2008 en 14 localidades de la costa peruana (1388 ha aproximadamente), mientras que los estudios de Guerra-Correa (2003) reportan que varían entre 257 287 individuos para la península de Mejillones en Chile.

El alto número de individuos de $S$. lorata encontrados en mayo del 2007 probablemente puedan explicarse por la llegada de individuos proveniente de otros lugares en la RNP luego de la culminación del periodo reproductivo. En este sentido, considerando este valor y la variación temporal en el número de individuos registrados, es recomendable continuar con los conteos mensuales, replicarlos en otros sectores de la RNP y en otros lugares fuera de esta área natural protegida para determinar el número de individuos y conocer más sobre el uso del hábitat de esta especie.

Considerando que la población global de $S$. lorata ha sido estimada por BirdLife International entre 1000 a 2499 individuos (BirdLife International, 2011), con los resultados de la presente investigación podemos precisar que en la península de Paracas se podría encontrar entre el $24.2 \%$ al $60.5 \%$ de la población mundial de esta especie.

Asimismo, los hallazgos de Murphy en 1920 (Murphy, 1936) sobre indicios de reproducción en la península de Paracas y las observaciones de Coker en 1907 (Coker, 1919) de abundantes individuos durante varios periodos del año en la bahía de Paracas sugieren que esta especie ha venido reproduciendose en la península de Paracas por lo menos desde inicios de 1900. Reportes de filopatría en esta especie (Amorós et al., 2010) también permiten sugerir el uso permanente del desierto de la península de Paracas como un sitio importante para la reproducción de esta especie.

Consideramos entre los depredadores potenciales de esta especie a Pseudalopex sp., "zorro"; Falco peregrinus, "halcón peregrino"; Buteo polyosoma, "aguilucho común” y Cathartes aura, "gallinazo cabeza roja”. Sin embargo, durante todo el periodo de estudio no se ha observado la muerte de algún individuo y/o consumo de huevos y/o pichones por parte de estos depredadores, pero sí un ataque sin éxito por parte del "aguilucho común" y evidencia de un ataque de "zorro" a uno de los nidos con presencia de huevos. Esto último concuerda con las observaciones hechas por Guerra-Correa (2003) en Mejillones, donde registraron el consumo de un huevo por Pseudalopex sp. Por el contrario, las amenazas de origen antrópico podrían afectar en mayor magnitud a $S$. lorata. Entre estas resalta el desarrollo de actividades no compatibles con la conservación del lugar, tales como la propuesta que existió para la extracción de sal (Vargas y Obando, 2008), y el tránsito vehicular desordenado por el interior de la colonia reproductiva.

El tránsito vehicular al interior de la colonia reproductiva sin un plan de señalización puede ocasionar riesgo de atropello de nidos con huevos y/o pichones, así como la pérdida de calidad del hábitat reproductivo por el aumento de las perturbaciones. La Tabla 1 muestra el riesgo de esta amenaza sobre los nidos de $S$. lorata; sin embargo, el número de trochas que atraviesan la península de Paracas y específicamente la colonia reproductiva de esta especie se incrementó luego del terremoto de Pisco (15 de agosto del 2007). Por consiguiente, se incrementaron las trochas cercanas a los nidos siendo mayor el riesgo de atropello de nidos y las perturbaciones en la zona de reproducción.

Adicionalmente a la importancia que la península de Paracas tiene para la conservación de $S$. lorata, debe considerarse que en este espacio también se encuentran lugares de reproducción, descanso y alimentación de otras especies de aves marinas y de orilla. Entre estas se cuenta con las colonias reproductivas de Oceanodroma markhami, "golondrina negra de la tempestad", especie de la cual se encontraron nidos activos en sectores descritos por Janhcke (1994); Haematopus palliatus "ostrero común” el cual se reproduce cerca de las orillas de playa; así como también Charadrius alexandrinus "chorlo nevado" y lugares de descanso de Calidris alba, "playerito blanco".

Las características de los nidos de $S$. lorata y Charadrius alexandrinus permiten determinar claramente a cuál de las dos especies corresponde, a pesar que en algunas oportunidades no se encontró la respectiva presencia de los adultos en sus nidos. Charadrius alexandrinus tiende a decorar sus nidos con presencia de piedras y/o restos de bivalvos (Figura 3a) mientras que los nidos de $S$. lorata consisten de una pequeña depresión sin mayor decoración (Figura 3c). En el caso de los huevos de Charadrius alexandrinus, estos son de color blanco con marcas negras a diferencia de los huevos de $S$. lorata que son de color pardo con presencia de marcas marrones (Amorós et al., 2010). También, el hallazgo de un nido de Charadrius alexandrinus a $1.5 \mathrm{~m}$ aproximadamente de un nido activo de $S$. lorata podría ser explicado por el beneficio reproductivo que los gaviotines le confieren a los chorlos, ya que los gaviotines suelen defender sus nidos $\mathrm{y}$ pichones mediante gritos de alarmas $y$ ataques ante perturbaciones en su zona de reproducción, tal como lo demostró Powell (2001) en California donde encontraron que el éxito de la nidada de Charadrius alexandrinus fue mayor para los individuos que nidificaron más cerca de un nido activo de Sterna 
antillarum. Sin embargo, requerimos de mayores estudios sobre estos aspectos para identificar un patrón característico.

La presencia del mayor número de nidos e individuos observados en la península de Paracas resalta la importancia de proteger este lugar para la conservación de $S$. lorata; sin embargo, también debe considerarse con igual importancia el desarrollo de estrategias de conservación en los otros lugares donde se ha reportado la especie (Tabla 2 y Tabla 3 ) de tal manera que estos esfuerzos pueden implementarse a lo largo de toda su distribución (Ecuador, Perú y Chile) y así garantizar la conservación de esta especie amenazada.

\section{Conclusiones.}

La península de Paracas constituye un lugar prioritario para la conservación de $S$. lorata, debido a que en esta localidad se ha encontrado el mayor número de nidos, así como también el mayor número de individuos observados respecto a otros lugares donde la especie se distribuye. Asimismo, en la península de Paracas se encontraría entre el $24.2 \%$ y el $60.5 \%$ de la población mundial reportada para esta especie.

La principal amenaza que afecta a $S$. lorata en la península de Paracas es de carácter antrópico tal como lo representa el tránsito vehicular que atraviesa su zona de reproducción.

La población de $S$. lorata se encuentra altamente amenazada en todo su ámbito de distribución y las investigaciones históricas muestran que su tamaño poblacional está disminuyendo. Por lo tanto, resulta particularmente importante apoyar los esfuerzos de conservación que se realizan al interior de esta área natural protegida y plantear estrategias que permitan mitigar las amenazas que la afectan. Asimismo, debe considerarse que para garantizar la conservación de $S$. lorata debe conservarse también los otros lugares donde la especie ha sido reportada. La implementación de acciones de conservación en Paracas requiere que estas tengan un enfoque participativo para involucrar a los principales actores en la mitigación de las amenazas que afectan a esta especie y así evitar la pérdida de calidad de su hábitat reproductivo.

\section{Agradecimientos.}

A Maria del Pilar Ramírez, Rachel Quispe, al personal de la RNP, Karin Obando, Liliana Ayala, Luis F. Felipe, Luis F. Cisneros, Luzdeli Cantoral, Sebastián Tapia, Ali Altamirano, Juan Guerra, Carlos A. Arnillas, Claudia Véliz, Carolina Tovar, Alessandro Catenazzi, Carlos Zavalaga, Carlos Guerra-Correa, Shaleyla Kélez, Fernando Angulo y Marta Williams.

Este estudio fue financiado con fondos del Programa de Becas para Investigación de Especies
Amenazadas "María Koepcke" de la Asociación Peruana para la Conservación de la Naturaleza APECO y Conservación Internacional - CI..

\section{Literatura citada.}

Angulo-Pratologo F. Shulenberg TS. \& Puse-Fernández E. 2010. Las aves de los humedales de Eten, Lambayeque, Perú. Ecología Aplicada 9(2):71-81

Amorós S. 2009. Importancia de la Península de Paracas (Ica, Perú) en la conservación del "Gaviotín Peruano" Sternula lorata (Philippi y Landbeck, 1981). Tesis para optar el título de Biólogo, Facultad de Ciencias, Departamento de Biología, Universidad Nacional Agraria La Molina, Lima.

Amorós S., Saravia P. \& Williams M. 2010. Biología Reproductiva de Sternula lorata, "gaviotín peruano", en la Reserva Nacional de Paracas (RNP), Ica - Perú. Revista Ecología Aplicada 9(2):125-132.

Amorós S. 2011. Indicios de nuevos sitios reproductivos del Gaviotín peruano, Sternula lorata (Charadriiformes, Laridae) en La Libertad, Perú, e implicancias para su conservación. The Biologist 9(2):177-192.

BirdLife International. 2011. Species factsheet: Sterna lorata. Consultado 17 de octubre del 2011. Disponible en http://www.birdlife.org.

Coker R. 1919. Habitats and economics relations of guano birds of Peru. Proceedings of Unites States National Museum. 56: 449 - 511

Chávez C. 2007. Las aves del santuario de conservación regional manglares de San Pedro de Vice, Sechura, Perú. Cotinga 27: 32 - 37

Chubb C. 1919. Notes on collections of birds in the British Museum, from Ecuador, Peru, Bolivia and Argentina. Ibis 61:1-55.

Duffy D., Hays C. \& Plenge M. 1984. The conservation status of Peruvian seabirds. ICBP Technical Publication No. 2: 245 - 259.

Decreto Supremo No 034-2004-AG. 2004. El Peruano. Año XXI - N 8859. Miércoles 22 de setiembre de 2004. Pág. 276853 - 276855. Consultado 1 de julio del 2009. Disponible en http://www.elperuano.com.pe/

Galarza N. 1968. Informe sobre estudios ornitológicos realizados en el laboratorio de la Puntilla (Pisco) en setiembre de 1965/66. Serie de Informes Especiales $N^{\circ}$ IM-31. Instituto del Mar del Perú.

Guerra-Correa C. 2003. Nidificación del Gaviotín Chirrío, Sterna lorata en Bahía Mejillones del Sur: Mitigación y manejo para la protección de las poblaciones locales. Corporación Nacional del Cobre de Chile, CODELCO FUREME. Informe 2002 Año 1. Antofagasta, Chile. 75 pp.

Guerra-Correa C. 2005. Fauna de Vertebrados, Flora y Vegetación de la Desembocadura del Río Loa. I y II Región de Chile. Universidad de Antofagasta - Centro Regional de Estudios y Educación Ambiental (CREA). Chile. 52 pp.

Harrison P. 1985. Seabirds an identification guide. Houghton Mifflin Company Boston. 448 pp.

INRENA. 2002. Plan Maestro 2003 - 2007 de la Reserva Nacional de Paracas. Pisco - Perú. 156 pp + anexos

Janhcke J. 1994. Distribución, situación poblacional y algunas características reproductivas de la golondrina de tempestad negra Oceanodroma markhami (Salvini 
1983) en la Reserva Nacional de Paracas, Perú. Tesis para optar el título de Biólogo. Facultad de Ciencias Departamento de Biología. Universidad Nacional Agraria La Molina. Lima - Perú. 87 pp + anexos.

Murphy R. C. 1936. Oceanic birds of South America. The Mc Millan Company, New York. 1245 pp.

Powell A. 2001. Habitat characteristics and nest success of Snowy plovers associated with California least tern colonies. The Condor 103:785-792

Rodríguez R. Editor. 1987. Manual de Técnicas de Gestión de Vida Silvestre. Wildlife Society. 4ta Edición. Versión en Español. 703 pp.

Rottmann J. \& González C. 1991. Gaviotín chico (Sterna lorata). Boletín informativo Unión de Ornitólogos de Chile 11:13

Senner N. 2006. First record of Long-billed Curlew Numenius americanus in Peru and other observations of Nearctic waders at the Virilla estuary. Cotinga 26: 39-42

Tello A., Engblom G., Merino P. \& Chalco J. J. 2005. Sterna lorata, situación en la costa central del Perú. Pp. 117 en Libro de resúmenes VI Congreso Nacional de Ornitología Chiclayo, Perú. Riva-Melofiro, F. y A. More, eds. Chiclayo, Perú.
Tello A., Quiñónez A. \& Merino P. 2008. Evaluación de la avifauna local y migratoria en los Humedales de Paraíso. Gerencia Regional Recursos Naturales y Gestión del Medio Ambiente. Huacho - Perú. 33 pp. Consultado 1 de julio del 2009. Disponible en: http://www.regionlima.gob.pe/gerencias/rrnn/estudios/e studio_avifauna_araiso.pdf

Vargas N. \& Obando C. 2008. Proyecto: Aprovechamiento Sostenible del Agua Marina para la Obtención de la Sal en Pozas de Cristalización en la Zona Norte Desierto de Pampa Lagunilla-RNP distrito de ParacasPisco. Corporación Salinera Paracas S.A.C.

Velarde D. Editor. 1998. Programa de Conservación y Desarrollo Sostenido de Humedales, Perú. Resultados de los Censos Neotropicales de Aves Acuáticas en el Perú 1992 - 1995. 154 pp.

Vilina Y. 1998. Breeding observations of the Peruvian Tern in Chile. Colonial Waterbirds 21 (1): 101-103.

Zavalaga C. B., Plenge M. \& Bertolero A. 2008. Nesting habitat and numbers of Peruavian Terns at five breeding sites in the central-southern coast of Peru. Ornitología Neotropical 19:587-594.

Zavalaga C. B., Harvesty J., Mori G., Chávez-Villavicencio C. \& Tello A. 2009. Current status of Peruvian Terns Sternula lorata in Perú: threats, conservation and research priorities. Bird Conservation International 19:175-186.

\section{ANEXOS}

Tabla 2. Máximo número de nidos de Sternula lorata “Gaviotín peruano” por sitio de reproducción en Perú y Chile

\begin{tabular}{|c|c|c|c|c|}
\hline Lugar (Coordenadas) & $\begin{array}{c}\mathrm{N}^{\circ} \text { máx. } \\
\text { nidos }\end{array}$ & $\begin{array}{l}\text { Fechas de } \\
\text { evaluación }\end{array}$ & Fuente & Observaciones \\
\hline
\end{tabular}

País: Perú

Pacasmayo (Dpto. La

Libertad), $7^{\circ} 24^{\prime}$ 'S, $79^{\circ} 35^{\prime} \mathrm{O}$

$12-13$
2008

C. Zavalaga y G. Mori en Zavalaga et al., 2009

Pampa de Urricape (Dpto. La Libertad), $7^{\circ} 32^{\prime} \mathrm{S}$, 1 $79^{\circ} 30^{\prime} \mathrm{O}$

Pativilca (Dpto. Lima), $10^{\circ} 41^{\prime} \mathrm{S}, 77^{\circ} 49^{\prime} \mathrm{W}$ Lima), $11^{\circ} 13^{\prime}-11^{\circ} 10^{\prime} \mathrm{S}$; $77^{\circ} 35^{\prime}-77^{\circ} 40^{\prime} \mathrm{O}$

\section{0 de marzo Amorós, 2011 2011}

M. Koepcke compilado por M. A. Plenge en Zavalaga et al., 2008

3 octubre 2003 febrero 2008
Tello et al., 2005 y Tello et al., 2008
Pacasmayo constituye el primer reporte de reproducción para el Perú; en esta localidad Murphy en 1920 encontró dos nidos con dos huevos cada uno y un nido con un pichón (Murphy, 1936).

Observaciones fuera del pico reproductivo. Se encontró un nido con presencia de dos huevos abandonados.

El 23 de enero del 2008 C. Zavalaga, G. Mori, J. Hardesty buscaron nidos pero no encontraron ninguno (Zavalaga et al., 2009).

Nidos hallados entre octubre noviembre del 2003. 
Puerto Viejo (Dpto. Lima), $12^{\circ} 34^{\prime} \mathrm{S}, 76^{\circ} 42^{\prime} \mathrm{O}$

Pampa Lechuza, Península de Paracas (Dpto. Ica), $13^{\circ} 53^{\prime} \mathrm{S}, 76^{\circ} 20^{\prime} \mathrm{W}$

Pampa Atenas - Cequión, Península de Paracas (Dpto. Ica), $13^{\circ} 51 ' \mathrm{~S}, 76^{\circ} 19^{\prime} \mathrm{O}$

Tres Hermanas - Yanyarina (Dpto. Ica), $15^{\circ} 27^{\prime} \mathrm{S}$, $75^{\circ} 03^{\prime} \mathrm{O}$

Mollendo (Dpto. Arequipa), $17^{\circ} 04^{\prime} \mathrm{S}, 71^{\circ} 58^{\prime} \mathrm{O}$
6

1969 a 1975

M. A. Plenge en Zavalaga et al., 2008

37 noviembre 2006 - mayo 2007

8 julio 2008

Amorós et al., 2010

3 26 enero 2008

J. Hardesty en Zavalaga et al., 2009

30

1969 a 1980

\section{R. A. Hughes en Duffy et al., 1984}

R. A. Hughes compilado por M. A. Plenge en Zavalaga et al., 2008
Observaciones semanales entre octubre a marzo

El lugar reproductivo fue abandonado en 1977 con el crecimiento urbano en zonas cercanas.

Temporada reproductiva 20062007.

En el conteo de nidos los autores solo consideran como nidos de Gaviotín peruano a aquellos que tuvieron presencia de huevos y/o pichones en su interior.

Fuera de temporada reproductiva (nidos con huevos abandonados)

Por lo menos observó 32 volantones.

También en Yanyarina, Zavalaga et al. (2008), encontraron 5 nidos el 11 de noviembre de 1997 y el 13 de diciembre del 2000, 70 individuos adultos.

Observaciones por lo menos una vez por mes

R. A. Hughes menciona cientos de parejas en la década de los 60 y alrededor de 12 parejas en 1982.

El lugar reproductivo fue abandonado en 1978 por perturbaciones humanas en la zona. Los números de nidos decrecieron de 30 en 1970-71 a 10 en 1973-74.

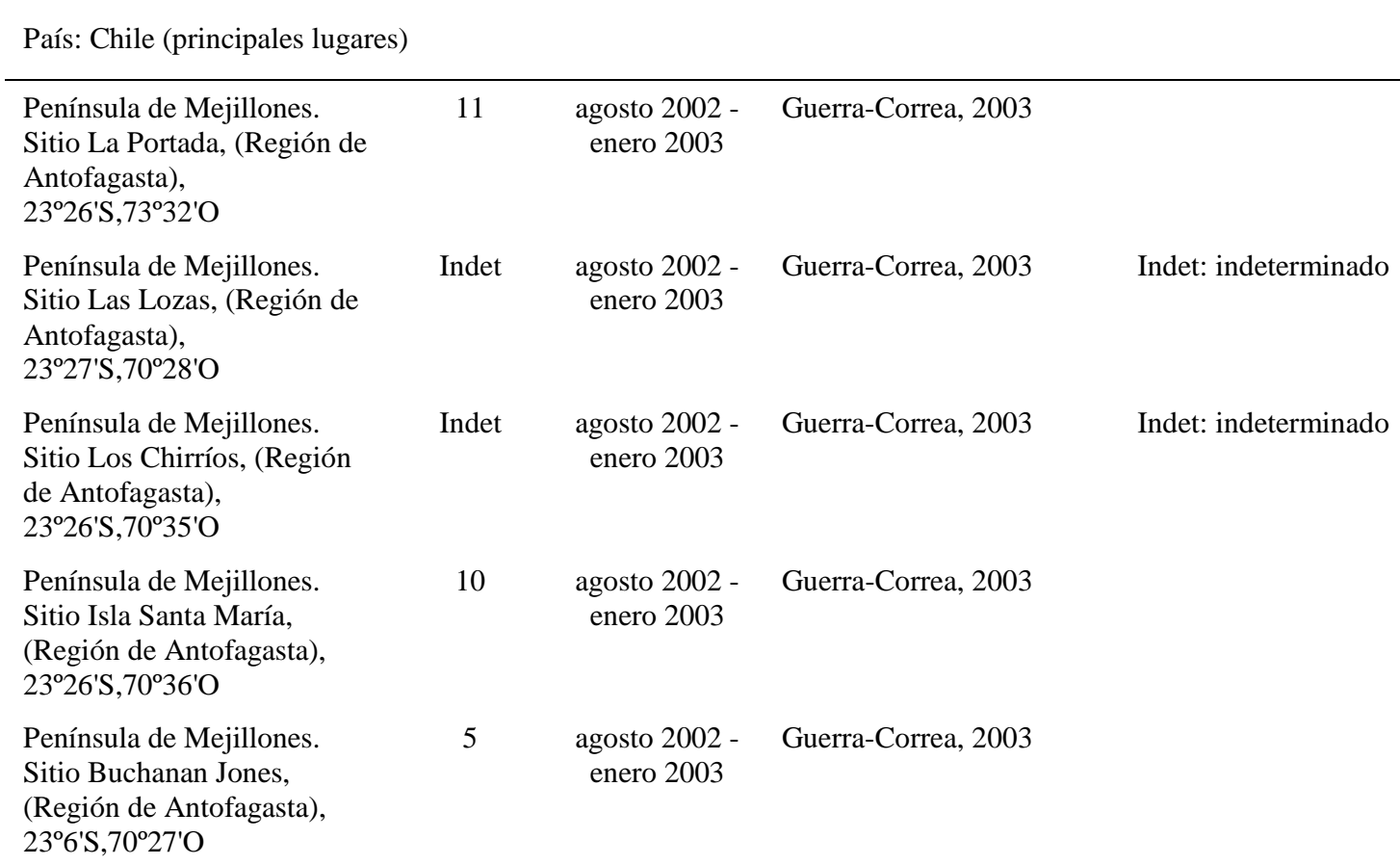


Península de Mejillones. Sitio Mejillones Industrial, (Región de Antofagasta), $22^{\circ} 599^{\prime} \mathrm{S}, 73^{\circ} 31^{\prime} \mathrm{O}$

Península de Mejillones. Sitio Chacaya - Itata,

(Región de Antofagasta), $22^{\circ} 53^{\prime} \mathrm{S}, 73^{\circ} 30^{\prime} \mathrm{O}$

Península de Mejillones. Sitio Hornitos - Yayes, (Región de Antofagasta), $22^{\circ} 54^{\prime} \mathrm{S}, 70^{\circ} 16^{\prime} \mathrm{O}$
35

agosto 2002 - Guerra-Correa, 2003

enero 2003

30

agosto 2002 - Guerra-Correa, 2003

enero 2003

Indet

agosto 2002 - Guerra-Correa, 2003 enero 2003
Indet: indeterminado

Tabla 3. Máximo número de individuos de Sternula lorata, "Gaviotín peruano”, por sitio de reproducción y/u observación en Ecuador, Perú y Chile.

\begin{tabular}{|c|c|c|c|c|}
\hline Lugar & $\begin{array}{l}\mathrm{N}^{\circ} \text { máx. } \\
\text { Individuos }\end{array}$ & $\begin{array}{l}\text { Fechas de } \\
\text { evaluación }\end{array}$ & Fuente & Observaciones \\
\hline
\end{tabular}

País: Ecuador

\begin{tabular}{|c|c|c|c|c|}
\hline $\begin{array}{l}\text { Isla Jambelí (Prov. El } \\
\text { Oro),32', } 80^{\circ} 08^{\prime} \mathrm{O}\end{array}$ & 176 & agosto 2011 & R. Ahlman com pers. & $\begin{array}{l}\text { En agosto del 2010, R. Ahlman } \\
\text { contó } 343 \text { individuos } \\
\text { (www.pbase.com/ahlman/terns), } \\
\text { coordenadas aproximadas. }\end{array}$ \\
\hline
\end{tabular}

País: Perú

\begin{tabular}{|c|c|c|c|}
\hline $\begin{array}{l}\text { Manglares de San Pedro } \\
\text { (Dpto. Piura), 531's, } \\
80^{\circ} 53^{\prime} O\end{array}$ & 37 & $\begin{array}{l}07 \text { agosto } \\
2010\end{array}$ & $\begin{array}{l}\text { F. Angulo, T. Valqui, A. } \\
\text { Garcia, L. Alza, R. Piana } \\
\text { y S. Crespo com pers. }\end{array}$ \\
\hline $\begin{array}{l}\text { Estuario de Virrilá (Dpto. } \\
\text { Piura), } \\
5^{\circ} 50^{\prime} S, 80^{\circ} 51^{\prime} \mathrm{O}\end{array}$ & 4 & julio 2007 & $\begin{array}{l}\text { C. Chávez en Zavalaga et } \\
\text { al., } 2009\end{array}$ \\
\hline
\end{tabular}

En julio del 2006, se observó a 6 juveniles que eran alimentados por sus padres (Chávez, 2007)

550'S, 8051'O

Observación a 4 km al Este de la orilla del mar.

También Senner (2006) menciona observaciones en agosto del 2004 en el estuario y Amorós (2009) indica el registró de un individuo adulto en agosto del 2006 a $20 \mathrm{~km}$ aproximadamente al interior del estuario desde el mar.

Isla Lobos de Tierra (Dpto. Lambayeque), $6^{\circ} 26^{\prime} \mathrm{S}, 80^{\circ}$ $51^{\prime} \mathrm{O}$

Humedales de Puerto Etén (Dpto. Lambayeque), 6 $55^{\circ}$ 's, 7954’O
100

junio 2006

A. García-Godos en Zavalaga et al., 2009

15

$\begin{array}{cl}28 \text { octubre } & \text { F. Angulo en Zavalaga et } \\ 2006 & \text { al., } 2009\end{array}$

TS. Schulenberg en Angulo et al. (2010) indican un registro de 40 individuos en julio de 1979.

La presencia de Sternula lorata para esta localidad han sido reportadas desde setiembre de 1899 por Chubb (1919), quien indica que realizó una colecta de 11 machos, 1 hembra, adultos e inmaduros en un lugar llamado "San Josicita” en Eten.

Incluye la observación de 1

Pampa de Río Seco (Dpto. La
Libertad), $7^{\circ} 12^{\prime} \mathrm{S}, 79^{\circ} 39^{\prime} \mathrm{O}$

7

21 marzo Amorós, 2011

2011 juvenil 


\author{
Pacasmayo (Dpto. La \\ Libertad), \\ $7^{\circ} 25^{\prime} \mathrm{S}, 79^{\circ} 35^{\prime} \mathrm{O}$
}

Pampa de Urricape (Dpto. La Libertad), $7^{\circ} 35^{\prime} \mathrm{S}, 7^{\circ} 28^{\prime} \mathrm{O}$

Huanchaco (Dpto. La

Libertad), 805'S, 7907’O

Punta Literas - Pativilca

(Dpto. Lima), 10³5'S, $77^{\circ}$

53 ’

Laguna Paraíso (Dpto. Lima),

$11^{\circ} 13^{\prime}-11^{\circ} 10^{\prime}$ 's; $77^{\circ} 35^{\prime}$ -

$77^{\circ} 40^{\prime} \mathrm{O}$

Isla Mazorca (Dpto. Lima), $11^{\circ} 22^{\prime} \mathrm{S}, 77^{\circ} 43^{\prime} \mathrm{O}$

Callao hasta $40 \mathrm{~km}$ aguas afuera, $12^{\circ} 05^{\prime} \mathrm{S}, 7^{\circ} 19^{\prime} \mathrm{O}$

Isla San Lorenzo (Dpto. Lima), $12^{\circ} 05^{\prime} \mathrm{S}, 77^{\circ} 12^{\prime} \mathrm{O}$

Puerto Viejo (Dpto. Lima), $12^{\circ} 34^{\prime} \mathrm{S}, 76^{\circ} 42^{\prime} \mathrm{O}$

Pampa Lechuza, Península de Paracas (Dpto. Ica), 135ㅗ's, 76² $19^{\prime} \mathrm{O}$

Tres Hermanas - Yanyarina (Dpto. Ica), $15^{\circ} 27^{\prime} \mathrm{S}, 75^{\circ} 03^{\prime} \mathrm{O}$

Mollendo (Dpto. Arequipa), $17^{\circ} 04^{\prime} \mathrm{S}, 71^{\circ} 58^{\prime} \mathrm{O}$

Humedales de Mejía (Dpto. Arequipa), $17^{\circ} 02^{\prime} \mathrm{S}, 72^{\circ} 00^{\prime} \mathrm{O}$

Humedales de Ite (Dpto.

Tacna),

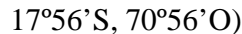

605

Quincenal entre

noviembre

2006 -

mayo 2007

32

26 enero del 2008

200

1969 a 1980

al., 2009 R. A. Hughes en Duffy et
al., 1984

R. A. Hughes compilado por M. A. Plenge en Zavalaga et al., 2008

M. A. Plenge en Zavalaga et al., 2008

1

30

2004

14 julio 2007
M. Ugarte-Lewis en

Zavalaga et al., 2009

C. Zavalaga en Zavalaga et al., 2009
El 12 al 13 de enero del 2008, C. Zavalaga y G. Mori observaron 7 individuos (Zavalaga et al., 2009)

El 20 de marzo del 2011 se observaron 13 adultos y 3 juveniles (Amorós, 2011).

Observaciones en el mar.

Observaciones en el mar.

Observaciones mensuales en el mar.

Observó por lo menos 12 individuos en un valle cercano a la cumbre de la isla. Por el comportamiento de las aves probablemente se encontraban anidando (coordenadas aproximadas).

Observaciones semanales entre octubre a marzo.

El lugar reproductivo fue abandonado en 1977 con el crecimiento urbano en zonas cercanas.

Datos de la temporada reproductiva 2006-2007.

Menciona que por lo menos observó 32 volantones.

Evaluaciones por lo menos una vez al mes. El lugar reproductivo fue abandonado en 1978 por perturbaciones humanas. 
SAMUEL AMORÓS Y PATRICIA SARAVIA

Ecol. apl. Vol. $11 \mathrm{~N}^{0}$ 2, pp. 47-57

País: Chile (principales lugares)

\begin{tabular}{|c|c|c|c|c|}
\hline $\begin{array}{l}\text { Desembocadura del río Loa } \\
\text { (Región de Tarapacá y } \\
\text { Región de Antofagasta), } \\
21^{\circ} 26^{\prime} \mathrm{S}, 7^{\circ} 03^{\prime} \mathrm{O}\end{array}$ & 7 & $\begin{array}{l}\text { setiembre } \\
2004\end{array}$ & Guerra-Correa, 2005 & Coordenadas aproximadas. \\
\hline $\begin{array}{l}\text { Península de Mejillones } \\
\text { ( } 8 \text { colonias reproductivas), } \\
\text { Región de Antofagasta }\end{array}$ & $257-287$ & $\begin{array}{l}\text { agosto } 2002 \\
\text { - enero } 2003\end{array}$ & Guerra-Correa, 2003 & Ver coordenadas en Tabla 2 \\
\hline $\begin{array}{l}\text { Playa La Rinconada (Región } \\
\text { de Antofagasta), } \\
23^{\circ} 27^{\prime} S, 70^{\circ} 29^{\prime} \mathrm{O}\end{array}$ & 12 & $\begin{array}{c}04 \\
\text { noviembre } \\
1989\end{array}$ & $\begin{array}{l}\text { Rottman y González, } \\
1991\end{array}$ & Coordenadas aproximadas. \\
\hline
\end{tabular}

${ }^{1}$ Afiliación cuando se realizó la investigación: Asociación Peruana para la Conservación de la Naturaleza -
APECO, Lima Perú. Afiliación actual: Centro para la Conservación Integral de los Ecosistemas Marinos del
Pacífico Este - ecOceánica, y APECO. Dirección de correspondencia del autor principal: samorosk@yahoo.com
${ }^{2}$ Reserva Nacional de Paracas. Servicio Nacional de Áreas Naturales Protegidas del Perú. 